\title{
Kinetics of Fertilization in the Sea Urchin Strongylocentrotus franciscanus: Interaction of Gamete Dilution, Age, and Contact Time
}

\author{
DON R. LEVITAN ${ }^{1,2, *}$, MARY A. SEWELL ${ }^{1,2}$ AND FU-SHIANG CHIA ${ }^{1}$ \\ 'Department of Zoology, University of Alberta, Edmonton, Alberta, Canada T6G 2E9; and \\ ${ }^{2}$ Bamfield Marine Station, Bamfield, British Columbia, Canada VOR IBO
}

\begin{abstract}
Determining fertilization success of free spawning organisms in the field requires knowledge of how eggs and sperm interact under varying encounter frequencies and durations. In the laboratory, we investigated the relative influence of sperm concentration, egg concentration, sperm-egg contact time, and sperm age on fertilization in the sea urchin Strongylocentrotus franciscanus. Our results indicated that sperm concentration, sperm-egg contact time, sperm age, and individual variability were sequentially the most important factors influencing fertilization success. Egg concentration was not significant over the range tested. A theoretical model of fertilization (Vogel-Czihak-Chang-Wolf model) was used to estimate the two rate constants of fertilization kinetics: the rate constant of sperm-egg encounter and rate constant of fertilization. This model explained $91 \%$ of the variation in fertilization success, provided estimates of the rate constants involved in fertilization, and indicated the proportion $(3 \%)$ of sperm-egg contacts that result in fertilization. Estimates of sperm swimming velocity and egg diameter were used to independently calculate the rate of spermegg encounter and confirm the predictions of the model. This model also predicts the non-significant effect of egg concentration on fertilization success found empirically.
\end{abstract}

\section{Introduction}

At least two approaches can be used to resolve questions concerning the fertilization success of free spawning organisms. The first is to collect field data during spawning events, and the second is to construct models based on

Received 13 May 1991; accepted 5 August 1991.

* Correspondence address: Department of Zoology, University of California, Davis, CA 95616-8755. laboratory observations of fertilization and field observations of water flow conditions. Because of inherent problems with both approaches, a combination of methodologies would provide the best understanding of the processes involved in field fertilization success and the most powerful predictive abilities. Problems with in situ measures of fertilization include experimental artifacts that may influence estimates of fertilization (Pennington, 1985; Yund, 1990; Levitan, 1991; and Levitan et al., in press) and the serendipitous nature of being able to collect relevant data during rare spawning events (Petersen, in press, Petersen et al., in press). Problems with the construction of fertilization models include gathering accurate estimates of water flow and turbulence during periods of spawning (for a model of fertilization in wave-swept shores see Denny and Shibata, 1989) and knowledge of how gametes interact under various encounter frequencies and durations. Here we investigate, in the laboratory, the interaction of gamete concentration, agc, and contact time in light of measures of sperm swimming velocity and egg size, and theoretical predictions of fertilization kinetics.

This is by no means the first attempt to describe fertilization success in the laboratory. There is a rich history of research on fertilization kinetics aimed at describing how the number of gametes, age of gametes, and other factors influence fertilization (e.g., Lillie, 1915; Rothschild and Swann, 1951; Hultin and Hagstrom, 1956; Brown and Knouse, 1973; Vogel et al., 1982; Pennington, 1985). These previous studies have documented that (1) fertilization is sensitive to sperm concentration and sperm age (references above), (2) fertilization is insensitive to egg concentration (Lillie, 1915), (3) fertilization is sensitive to the time of eggs spend in a sperm solution (Rothschild and Swann, 1951) and (4) fertilization kinetics should be 
a function of gamete concentration, sperm age, sperm velocity, and egg size (however, the dependence of fertilization on egg size and sperm velocity was predicted and not tested empirically-Vogel et al., 1982). These conclusions are crucial to our understanding of in situ fertilization (e.g., Denny, 1988; Denny and Shibata, 1989). However, no study to date has addressed these factors simultaneously under the range of values normally encountered in nature, or has used estimates of sperm velocity and egg size to predict rates of fertilization.

In this study we first address four parameters of gamete kinetics and their interaction: sperm concentration, sperm age, sperm-egg contact time (the time an egg spends at a particular sperm concentration), and egg concentration. Second, we use a fertilization kinetics model (Vogel $e t$ al., 1982 - see below) to predict the rate constants of fertilization and sperm-egg encounter frequency. Third, we test the model's predictions of these rate constants against empirical measures of egg size and sperm velocity. Finally, we use the fertilization kinetics model to evaluate the relative sensitivity of fertilization to sperm and egg concentrations normally encountered in nature. This knowledge about the kinetics of fertilization will enhance the accuracy of future theoretical models, and provide insight into the interpretation of empirical field data.

\section{The Vogel-Czihak-Chang-Wolf model}

Vogel et al. (1982) proposed a fertilization kinetics model that predicts fertilization based on sperm and egg concentration, the half-life of sperm ' $\tau$ ' and two rate constants $\beta_{0}$ (of sperm-egg contact, based on egg cross-sectional area and sperm swimming velocity) and $\beta$ (of fertilization, based on sperm-egg contact and the fertilizability of the egg). This model assumes that sperm attach to the first egg they contact regardless of whether fertilization takes place.

The Vogel-Czihak-Chang-Wolf (VCCW) model is described by the following equation (Vogel et al., 1982; $\mathrm{p}$. 203):

$$
\Phi \propto=1-\exp \left(-\frac{\beta \mathrm{S}_{0}}{\beta_{0} \mathrm{E}_{0}}\left(1-\mathrm{e}^{-\beta_{0} \mathrm{E}_{0} r}\right)\right)
$$

Where: $\quad \mathrm{S}_{0}=$ Sperm concentration $(\# / \mu \mathrm{l})$

$$
\mathrm{E}_{0}=\text { Egg concentration }(\# / \mu \mathrm{l})
$$

The ratio of $\beta / \beta_{0}$ is either the fertilizable area of the egg, the proportion of sperm able to fertilize an egg (Vogel et al., 1982), or the number of sperm contacts needed to allow for the penetration of a single spermatozoan. Vogel et al. (1982) suggest the first explanation, although these alternate hypotheses have not been directly tested.

Vogel et al. (1982) estimated $\beta$ and $\beta_{0}$ for the urchin Paracentrotus lividus. They observed ' $\tau$ ' to be $1500 \mathrm{~s}$ (they did not account for changes in ' $\tau$ ' as sperm concentration varied-see results) and using a nonlinear model determined the best fit of $\beta$ and $\beta_{0}$ to be $3.8 \times 10^{-6}$ and 3.3 $\times 10^{-4} \mathrm{~mm}^{3} / \mathrm{s}$, respectively. Vogel et al. suggest that because $\beta / \beta_{0}$ is approximately 0.01 , only $1 \%$ of the egg surface is fertilizable. This value is critical to the low fertilization predicted by Denny and Shibata (1989) in their model of fertilization success on wave swept shores.

\section{Materials and Methods}

\section{General}

Experiments were conducted between 27 February and 8 May 1990 at the Bamfield Marine Station on the west coast of Vancouver Island, British Columbia, Canada. All urchins were gathered from the mouth of Bamfield Inlet $\left(48^{\circ} 50^{\prime} 30^{\prime \prime} \mathrm{N}, 125^{\circ} 08^{\prime} \mathrm{W}\right)$, at a depth of $9 \mathrm{~m}$, from rock and cobble substrata. Animals were maintained in flowing seawater tables at $12^{\circ} \mathrm{C}$ prior to experiments. Gametes were collected by inducing urchins to spawn with a $5 \mathrm{ml}$ injection of $0.55 \mathrm{M} \mathrm{KCl}$. Sperm were obtained by inverting male urchins over a glass finger bowl. Sperm were kept "dry" and cool at $12^{\circ} \mathrm{C}$ until needed for experiments. Eggs were obtained by inverting female urchins over a large glass finger bowl filled with $1 \mu \mathrm{m}$ filtered seawater. Eggs and all experiments were maintained at $12^{\circ} \mathrm{C}$ in a running seawater table.

Sperm concentration in each experiment was determined by fixing $5 \mathrm{ml}$ of a $10^{2}$ dilution of dry sperm with five drops of $100 \%$ formalin. At this dilution, sperm counts were made using a hemocytometer (8 replicate counts of $2.5 \times 10^{-7} \mathrm{ml}$ sub-samples). Eggs were collected with a $10 \mathrm{ml}$ pipette and then diluted until there were between 6000 and 6500 eggs per $\mathrm{ml}$ in the stock egg suspension. For all experimental treatments, three replicates were conducted. In all but the preliminary sperm dilution experiment, each replicate used a different male and female. In the preliminary study, the replicates were from the same male and female. In all but the sperm-egg contact time experiments, eggs and sperm were placed into small vials, swirled for $15 \mathrm{~s}$ and the degree of fertilization assessed after $3 \mathrm{~h}$. The percentage of eggs fertilized was estimated, using a compound microscope, by the presence of a fertilization membrane or later cleavage stages in 100 undamaged eggs randomly sampled from each experimental treatment.

For statistical analysis, an arcsine transformation was performed on all percent fertilization data. An analysis of covariance was used for differences in the percentage of eggs fertilized in the sperm age, sperm-egg contact time, and egg concentration experiments. Sperm concentration (log transformed) was the covariate. These initial experiments establish the range of responses used in the multifactorial experiment and most importantly provide data 
for the fertilization kinetics model. In the multifactorial experiment, we used a step-wise regression to analyze the contribution of each factor in explaining variation in fertilization success and an analysis of covariance to analyze the variation among urchins in gamete quality.

\section{Sperm dilution experiment}

To determine the range of sperm concentration over which fertilization success declines from 100 to $0 \%$, we conducted a preliminary dilution experiment. A $1 \mathrm{ml}$ aliquot of a diluted egg suspension (6000 to 6500 eggs per $\mathrm{ml}$ ) was placed in a small vial with $8 \mathrm{ml}$ of filtered seawater. Fresh "dry" sperm were diluted in a series of 10, 10-fold dilutions. A $1 \mathrm{ml}$ aliquot from one of these sperm suspensions was then placed into the vial, to bring the final volumes to $10 \mathrm{ml}$ ( 10 dilutions $\times 3$ replicates $=30$ vials $)$.

\section{Sperm age experiment}

We assessed the influence of sperm age on fertilization using six levels of age $(0,10,20,40,80$, and $160 \mathrm{~min})$ over six levels of dilution $\left(10^{2}, 10^{4}, 10^{5}, 10^{6}, 10^{6.5}\right.$, and $10^{7}$ ). The range of dilutions was determined from the results of the previous experiment. A $1 \mathrm{ml}$ aliquot of a dilute sperm suspension was placed in a vial with $8 \mathrm{ml}$ of filtered seawater. A $1 \mathrm{ml}$ aliquot of a dilute egg suspension was added to the vial at the appropriate time: at $0,10,20,40$, 80 , and $160 \mathrm{~min}$ after the sperm were diluted ( 6 dilutions $\times 6$ times $\times 3$ replicates $=108$ vials). Although both sperm and eggs were aged in this experiment, previous research (e.g., Pennington, 1985) has shown that sperm are much more sensitive than eggs to age effects. Nevertheless, assuming synchronous spawning, sperm and eggs would age simultaneously in the field.

\section{Sperm-egg contact time experiment}

The influence of the duration of sperm contact on fertilization success was assessed by manipulating the time eggs were in contact with a diluted suspension of sperm. A $1 \mathrm{ml}$ aliquot of a dilute egg suspension was pipetted into a plastic cylinder $(66 \mathrm{~mm}$ in diameter and $30 \mathrm{~mm}$ deep with $35 \mu$ mesh Nitex covering the base). The cylinder was then placed in a petri dish filled with filtered seawater. Sperm were diluted into six concentrations (dilutions of: $10^{2}, 10^{3}, 10^{4}, 10^{4.5}, 10^{5}$, and $10^{6}$ ) each to a volume of 200 $\mathrm{ml}$. The egg-filled cylinder was placed in a clean petri dish and then $40 \mathrm{ml}$ of sperm (from one of the dilutions) were discharged from a syringe into the cylinder. The sperm remained in contact with the eggs for one of four time periods $(0.5,2,8$, and $32 \mathrm{~min})$. The container was then rinsed with filtered seawater to remove excess sperm, and placed back into the petri dishes filled with filtered seawater $(6$ dilutions $\times 4$ contact times $\times 3$ replicates $=72$ containers).

\section{Egg concentration experiment}

This experiment investigated how egg concentration influences fertilization over a range of sperm dilutions. We placed either a $1,2,4$, or $8 \mathrm{ml}$ aliquot of a dilute egg suspension ( 6000 to 6500 eggs per $\mathrm{ml}$ ) into a vial and then added a $1 \mathrm{ml}$ aliquot of a dilute sperm suspension (dilutions of either $10^{3}, 10^{5}$, or $\left.10^{7}\right)$ and filtered seawater to a final volume of $10 \mathrm{ml}$ ( 3 sperm dilutions $\times 4$ cgg dilutions $\times 3$ replicates $=36$ vials).

\section{Sperm-egg contact time, sperm age, and sperm dilution experiment}

In this experiment, three factors were varied simultaneously. Egg concentration was not manipulated because it was not significant over the range tested (see results). This experiment had four levels of sperm age $(5,15,25$, and $35 \mathrm{~min}$ ), four levels of sperm-egg contact time (7, 15, 30 , and $60 \mathrm{~s})$, and five dilutions $\left(10^{2}, 10^{3}, 10^{4}, 10^{5}\right.$, and $10^{6}$ ). The experimental protocol was identical to the sperm-egg contact time expcriment, with the exception of the $7 \mathrm{~s}$ contact time trials. In these trials, the containers did not sit in the sperm solution since the time needed to discharge the $40 \mathrm{ml}$ of sperm solution was $7 \mathrm{~s} \mathrm{(4} \mathrm{sperm}$ ages $\times 4$ contact times $\times 5$ dilutions $\times 3$ replicates $=240$ containers).

\section{Sperm velocity}

We estimated sperm swimming velocity by analyzing slow motion videotapes of sperm at a concentration of $1.50 \times 10^{7} / \mathrm{ml}$. Sperm were diluted and immediately placed in a depression slide on a cooled stage of a compound microscope and video taped at $400 \times$. The video recorded time with an internal stop-watch. Sperm swimming was analyzed by tracing individual spermatozoa on acetate sheets attached to a video monitor. Distances were measured with a graphics tablet and velocities calculated by dividing distance moved by the elapsed time.

\section{Results}

\section{Sperm dilution experiment}

High percent fertilization was noted at the $10^{2}$ dilution $\left(4.7 \times 10^{7} \mathrm{sperm} / \mathrm{ml}\right)$ and showed a slight decline until the $10^{6}\left(4.7 \times 10^{3}\right.$ sperm $\left./ \mathrm{ml}\right)$ dilution when fertilization decreased to $18 \%$ (Fig. 1). No fertilization was observed beyond the $10^{8}$ dilution ( $47 \mathrm{sperm} / \mathrm{ml}$ ).

For this, and in all other experiments, sperm in the $10^{2}$ dilution were still actively swimming after $3 \mathrm{~h}$ and development was generally halted with a raised fertilization membrane. The halted development at this high sperm concentration was probably due to polyspermy. At other dilutions, sperm movement was not detected after $3 \mathrm{~h}$, 


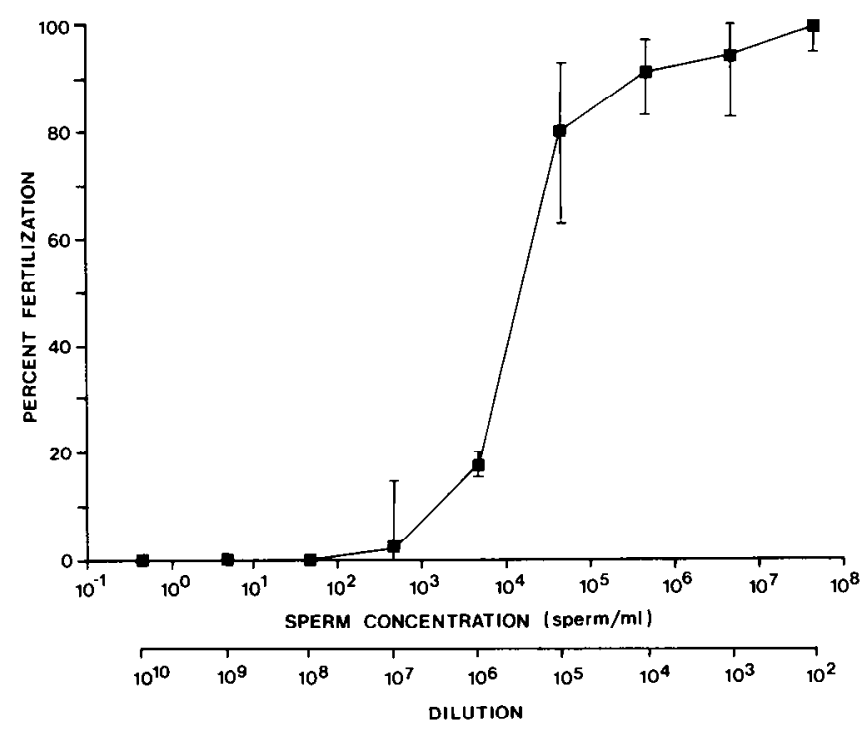

Figure 1. Fertilization as a function of sperm concentration $(\# / \mathrm{ml})$ in Strongylocentrotus franciscamus. Percent data were arcsine transformed, the mean and $95 \% \mathrm{CI}$ are plotted after back transformation $(\mathrm{n}=3)$. Sperm concentration estimated from hemocytometer counts at the $10^{2}$ dilution $\left(4.7 \times 10^{7}\right.$ sperm $\left./ \mathrm{ml}\right)$.

and development seemed to be progressing normally (e.g., developmental times consistent with Strathmann, 1987).

\section{Sperm age experiment}

The results indicated a highly significant effect of sperm age and sperm concentration on fertilization success (ANCOVA, $P<0.0001$ for both the covariate of sperm concentration and the main effect of sperm age). Figure 2 is a three-dimensional contour plot of fertilization as a function of sperm age and sperm dilution. Sperm age had little influence on fertilization at the highest sperm concentrations. In fact, sperm at the $10^{2}\left(9.85 \times 10^{7}\right.$ sperm/ $\mathrm{ml}$ ) dilution were still active after $7 \mathrm{~h}$. A 10-fold dilution of this aged $(7 \mathrm{~h})$ concentration resulted in nearly $100 \%$ fertilization. Sperm age had the most influence on fertilization at the $10^{5}$ dilution $\left(9.85 \times 10^{7} \mathrm{sperm} / \mathrm{ml}\right)$. At greater sperm dilutions, the influence of sperm age lessened due to an overall decrease in fertilization.

\section{Sperm-egg contact time}

The results indicated a highly significant effect of spermegg contact time and sperm concentration on fertilization success (ANCOVA, $P<0.0001$ for both covariate of sperm concentration and the main effect of sperm-egg contact time). Figure 3 is a three-dimensional contour plot of fertilization as a function of sperm-egg contact time and sperm dilution. Sperm-egg contact time had the greatest influence on fertilization at the intermediate dilution of $10^{4.5}\left(3.83 \times 10^{5} \mathrm{sperm} / \mathrm{ml}\right)$. At lesser or greater



Figure 2. Fertilization as a function of sperm age and sperm dilution in Strongylocentrotus franciscanus. Mean percent fertilization plotted, error bars were omitted for clarity. Because sperm concentration varied slightly between replicates, sperm dilution was plotted for clarity.

sperm dilutions, the influence of sperm contact time on fertilization decreased slightly.

Sperm-egg contact time influences fertilization most between the 0.5 and $2 \mathrm{~min}$ interval. This result suggests that sperm-egg contact time may be important at even finer time intervals. Thus, in the multi-factorial experiment we chose intervals of $7,15,30$, and $60 \mathrm{~s}$.

\section{Egg concentration}

The results indicated no significant effect of egg concentration, but a highly significant effect of sperm con-

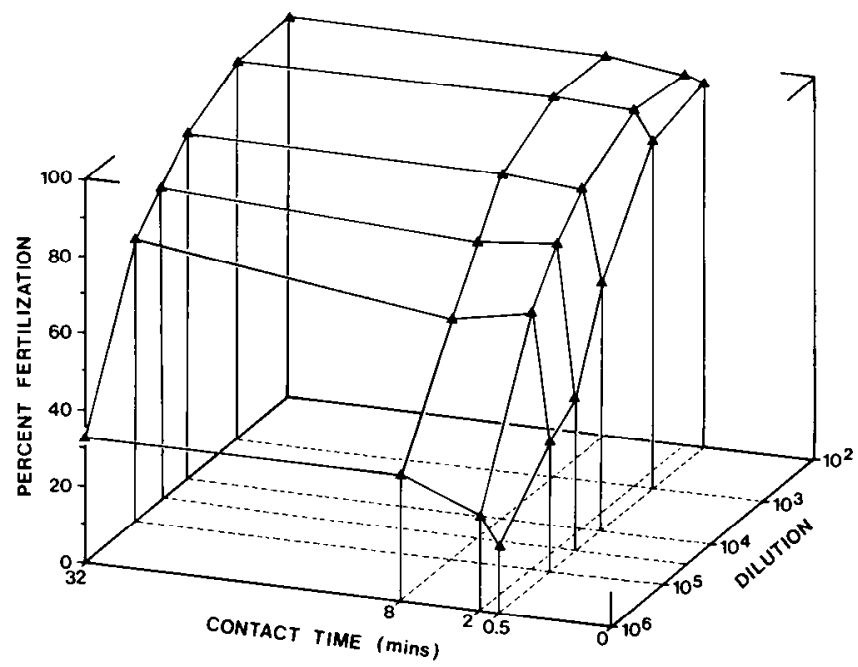

Figure 3. Fertilization as a function of sperm-cgg contact time and sperm dilution in Strongylocentrotus franciscanus. Mean percent fertilization plotted, error bars were omitted for clarity. Because sperm concentration varied slightly between replicates, sperm dilution was plotted for clarity. 
centration on fertilization success (ANCOVA, $P<0.0001$ for covariate of sperm concentration and $P>0.25$ for main effect of egg concentration). Figure 4 is a three-dimensional contour plot of fertilization as a function of egg concentration and sperm dilution.

\section{Sperm-egg contact time, sperm age, sperm dilution}

A forward step-wise regression analysis tested percent fertilization (arcsine transformed) with the following variables: sperm-egg contact time, sperm age, and sperm concentration ( $\log$ transformed). All variables were highly significant $(P<0.0001)$ with sperm dilution (Fig. 5A), sperm-egg contact time (Fig. 5B) and sperm age (Fig. 5C) explaining cumulatively $61.8,67.6$, and $70.1 \%$ of the variation. The regression generated from these results is:

$$
\mathrm{F}=\mathrm{S}(0.262)+\mathrm{T}(0.006)+\mathrm{A}(-0.007)-0.846
$$

Where: $\quad F=$ Percent fertilization (arcsine transformed)

$\mathrm{S}=$ Sperm concentration (sperm $/ \mathrm{ml}, \log$ transformed)

$\mathrm{T}=$ Sperm-egg contact time (s)

$\mathrm{A}=$ Sperm age $(\mathrm{min})$

With $c a .6250$ eggs

The remaining $30 \%$ of the variation can be attributed to three factors; within and between individual variation in gamete quality, and using linear or log transformed approximations for the relationships described above. The first two factors were addressed with an analysis of covariance used to compare the percentage of eggs fertilized (arcsine transformed), with gamete variability (individual male and female used for a series of experiments) as the

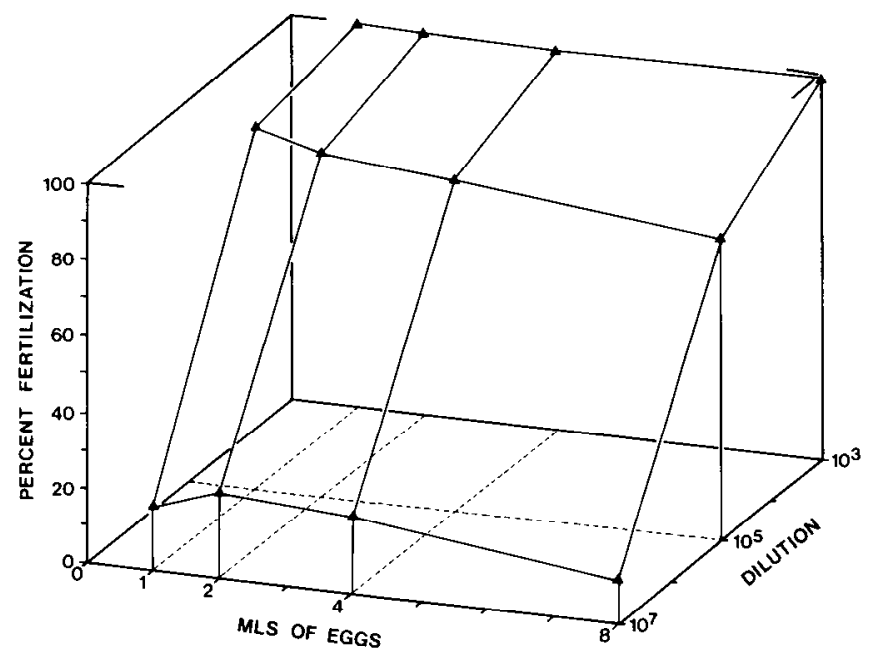

Figure 4. Fertilization as a function of mls of eggs ( $c a$. 6000-6500 eggs $/ \mathrm{ml}$ ) in $10 \mathrm{ml}$ volume and sperm dilution in Strongylocentrotus franciscanus. Mean percent fertilization plotted, error bars were omitted for clarity. Because sperm concentration varied slightly between replicates, sperm dilution was plotted for clarity.
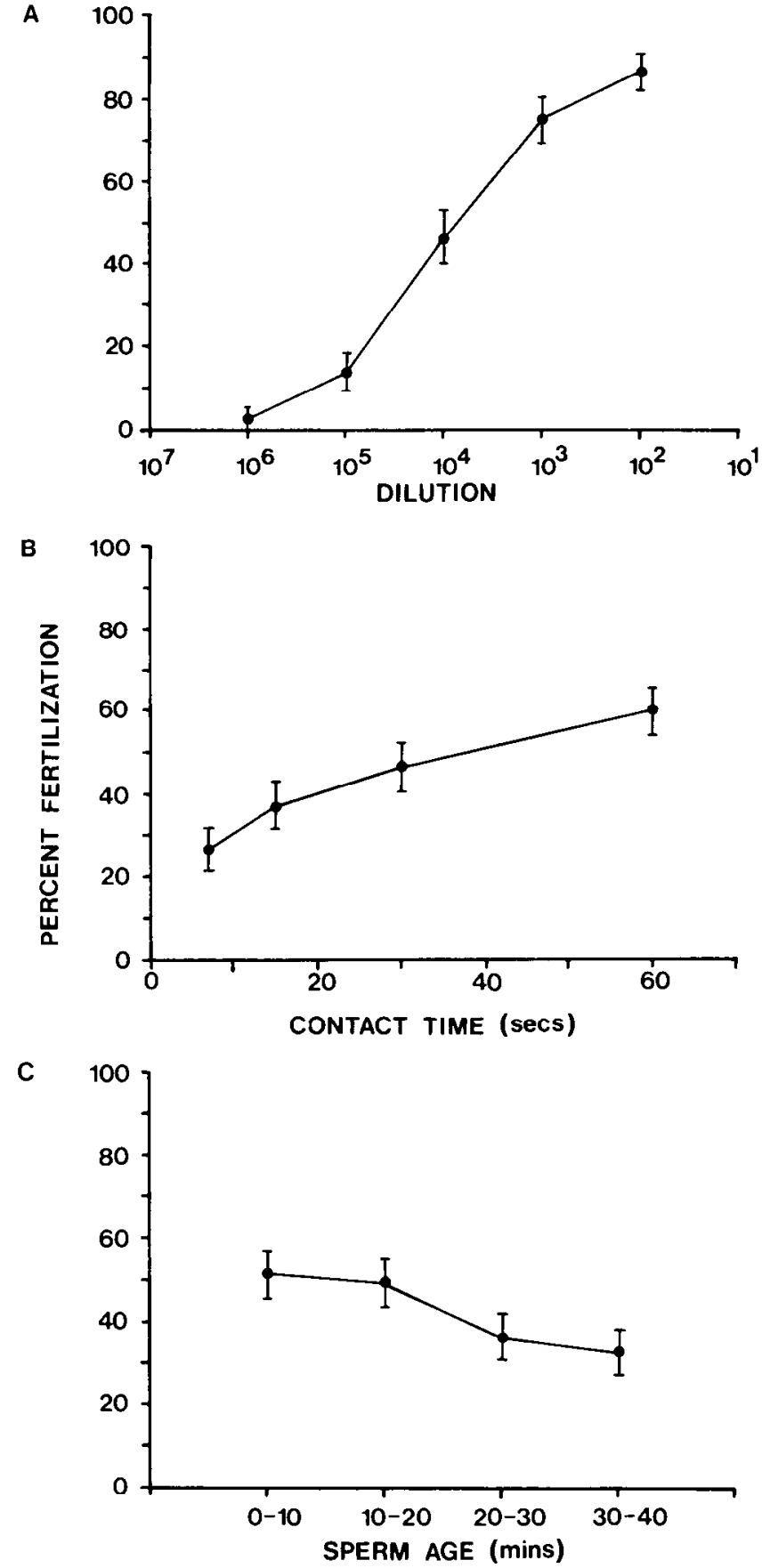

Figure 5. Fertilization in the multifactorial experiment; Strongylocentrotus franciscanus. Percent data were arcsine transformed, the mean and $95 \% \mathrm{CI}$ are plotted after back transformation ( 3 replicates, 5 dilutions, 4 sperm-egg contact times, and 4 sperm ages). A) Sperm dilution, B) Sperm-egg contact time, C) Sperm age.

main effect and sperm concentration (sperm $/ \mathrm{ml}$, log transformed), sperm-egg contact time (s) and sperm age (min) as covariates. All factors were significant; there were differences in the lcvel of fertilization between gametes from individual urchins $(P<0.0001$ for all covariates and 


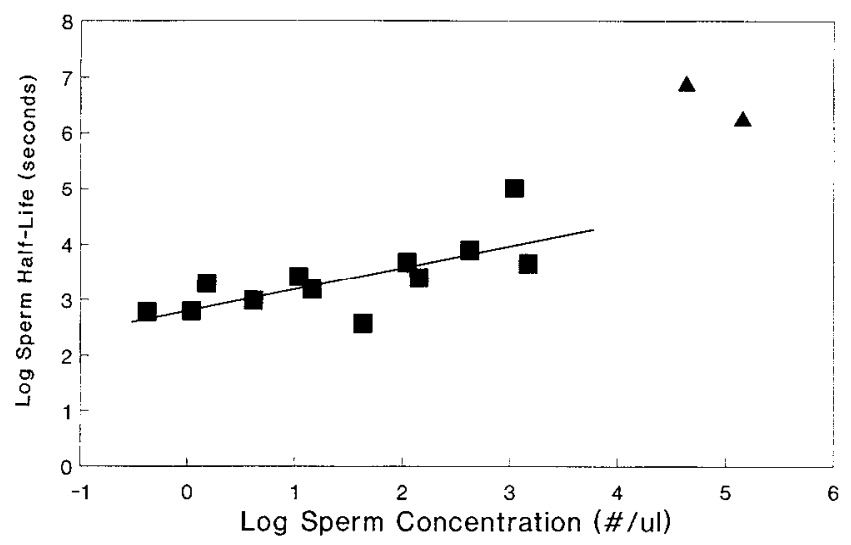

Figure 6. Sperm half-life (' $\tau$ '; $\log$ transformed) as a function of sperm concentration (log transformed) in Strongylocentrotus franciscanus. Each datum represents the sperm age when fertilization dropped to $50 \%$ of initial fertilization when sperm was freshly diluted. Triangle symbols represent sperm age trials where fertilization never dropped to $50 \%$ of initial fertilization. These noints were calculated beyond the range of the experiment and were not included in the regression equation (Log sperm half-life $(\mathrm{s})=[\log$ sperm concentration $(\# / \mu \mathrm{l}) \times 0.391]+2.818, \mathrm{R}^{2}$ $=0.51, \mathrm{n}=12$ ).

the main effect). The remaining problem of assuming linear or log-linear relations between the variables has been addressed theoretically by Vogel et al. (1982).

\section{The Vogel-Czihak-Chang-Wolf model}

We filted a subset of our data, using only manipulations with fresh gametes $(n=199)$, to the VCCW model using their equation "14" (Vogel et al., 1982; p. 203) which substitutes ' $t$ ' (the sperm-egg contact time) with ' $\tau$ ' (the sperm half-life). However, when ' $t$ ' exceeded ' $\tau$ ', we uscd the latter value, because higher values of ' $t$ ' would overestimate the vitality of the sperm.

We calculated values of ' $\tau$ ' by fitting the results of the sperm-age experiment at each dilution separately to an exponential regression equation. For each equation, the half-life was calculated from the predicted time when fertilization was half the initial value at time " 0 ." These halflife values were then plotted as a function of sperm concentration (log transformed, Fig. 6) and a linear regression was performed to predict ' $\tau$ ' values for the range of sperm concentrations used in the analysis.

To test the VCCW model we iterated the Marquardt method (the Gauss and Dud methods gave similar results) of non-linear regression (SAS statistical program) to find the best fitted values of $\beta$ and $\beta_{0}$ for our values of sperm

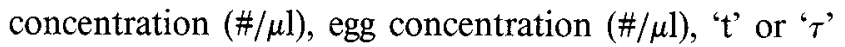
(s), and the observed fertilization ratio.

The model of Vogel et al. (1982) appears to be quite robust as it explained $91 \%$ of the variation in fertilization in Strongylocentrotus franciscanus (in spite of the unexplained individual variation in gamete quality). The rate constant of fertilization $(\beta)$ was estimated to be 6.044 $\times 10^{-5} \mathrm{~mm}^{3} / \mathrm{s}\left(\mathrm{SE}=1.072 \times 10^{-5}\right)$ and the rate constant of sperm-egg encounter $\left(\beta_{0}\right)$ was estimated to be 1.761 $\times 10^{-3} \mathrm{~mm}^{3} / \mathrm{s}\left(\mathrm{SE}=6.971 \times 10^{-4}\right)$. Both these estimates are one order of magnitude larger than estimated for Paracentrotus lividus by Vogel et al. (1982). The ratio of $\beta / \beta_{0}$ is calculated to be 0.0343 . This ratio is about three times higher than the ratio estimated by Vogel et al. (1982) and used by Denny and Shibata (1989) to predict fertilization in the field. Use of this ratio would therefore increase Denny and Shibata's predictions of in situ fertilization by a factor of threc (from approximately 1 to $3 \%$ in highly turbulent water).

An independent estimate of $\beta_{0}$ can be made from the following equation (Vogel et al., 1982; p. 208):

$$
\beta_{0}=\nu \times \sigma_{0}
$$

Where: $\quad \nu=$ Mean spermatozoan velocity $(\mathrm{mm} / \mathrm{s})$ $\sigma_{0}=$ Egg cross-sectional area $\left(\mathrm{mm}^{2}\right)$

We analyzed a total of 116 spermatozoa from 4 urchins (Fig. 7). There were significant differences in swimming velocity between individual urchins (ANOVA, $P<0.01$ ). These differences and perhaps similar differences in egg quality are possible reasons for individual variance in fertilization success. The mean sperm velocity was 0.149 $\mathrm{mm} / \mathrm{s}(\mathrm{SE}=0.0113)$. Using this estimate of swimming velocity and egg cross-sectional area of $0.014314 \mathrm{~mm}^{2}$ (egg diameter $0.135 \mathrm{~mm}$; pers. obs., Strathmann, 1987), $\beta_{0}$ was calculated to be $2.13 \times 10^{-3}\left(\mathrm{SE}=1.61 \times 10^{-3}\right)$. There was no significant difference between this estimate of $\beta_{0}$ and our estimate from the VCCW model (Student's ' $\mathrm{t}$ ' $=0.075, P>0.9$ ).

Vogel et al. (1982) did not measure sperm swimming directly, they estimated velocity $(\nu)$ by solving equation (2) using their estimates of $\beta_{0}$ and egg diameter. Their estimate for Paracentrotus lividus was $0.040 \mathrm{~mm} / \mathrm{s}$. Gray (1955) measured sperm swimming velocity directly for Psammechinus miliaris and found rates of $0.190 \mathrm{~mm} / \mathrm{s}$. Gray also reviews studies of several other echinoid species

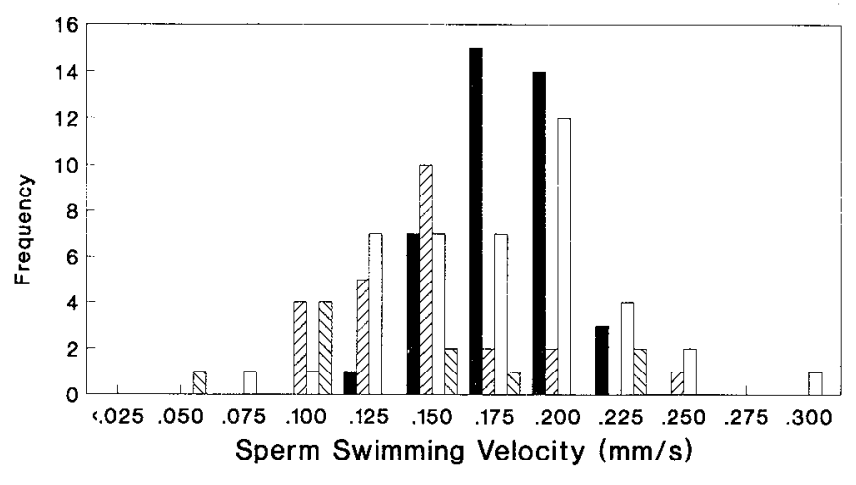

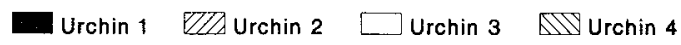

Figure 7. Frequency distribution of Strongylocentrotus franciscanus sperm swimming velocity. 
and reports rates of between 0.120 and $0.190 \mathrm{~mm} / \mathrm{s}(1955$ and references within). Because we found significant differences between individuals, it should not be surprising to find differences between species.

\section{Sensitivity of egg concentration to fertilization}

Because fertilization is a result of sperm-egg encounters, it seems intuitive that egg concentration should be important to fertilization success, yet our empirical results suggest otherwise. To evaluate the sensitivity of fertilization success to egg concentration, we again iterated the Marquardt method of non-linear regression to find the best fitted values of $\beta$ and $\beta_{0}$. This time we chose a subset of our data using only experiments with fresh gametes and constant egg concentrations $(n=40)$, to predict what fertilization should be in our egg concentration experiment. The empirical data indicated that the greatest effect of changes in egg concentration was a non-significant decrease of $4.9 \%$ between the lowest and highest egg concentration at the lowest sperm concentration. The VCCW model, using the subset of the data, predicted a decrease of only $6.3 \%$ over the same range of gamete concentration. There was no significant difference between the observed and predicted values (Student's ' $t$ ' $=0.492, P>0.5$ ). Figure 8 is a three-dimensional contour graph of fertilization as a function of both sperm and egg concentration, using the original data set $(n=199)$ in the VCCW model. Clearly, it predicts the insensitivity of the fertilization process to egg concentration. Only when the concentration was over 100 eggs per $\mu 1$ was there a slight impact on fertilization. Because eggs of $S$. franciscanus are extruded at a concentration of $325 / \mu$ l (range $169-499 / \mu \mathrm{l}, \mathrm{n}=5$ urchins, subsample counts), and rapidly diluted in seawater, egg concentration would only be important to fertilization success within the first few seconds of release.

\section{General discussion}

Sperm concentration had the greatest effect on fertilization followed by sperm-egg contact time and sperm age. We found no significant effect of egg concentration on fertilization. Part of the reason for the order of importance was associated with the relative range of each factor examined (but, see above for egg concentration). Sperm concentration was varied over 100,000 -fold, contact time was varied 64-fold in the first and 8-fold in the multi-factorial experiment, and sperm age was varied 160 fold in the first and 4-fold in the multifactorial experiment. Ranges were chosen based on results from the single factor results and our estimates on the probable interactions in the field (see below).

Sperm dilutes rapidly within a few meters from a sperm source (modelled by Denny, 1988; Denny and Shibata, 1989; empirical evidence by Pennington, 1985; Yund,

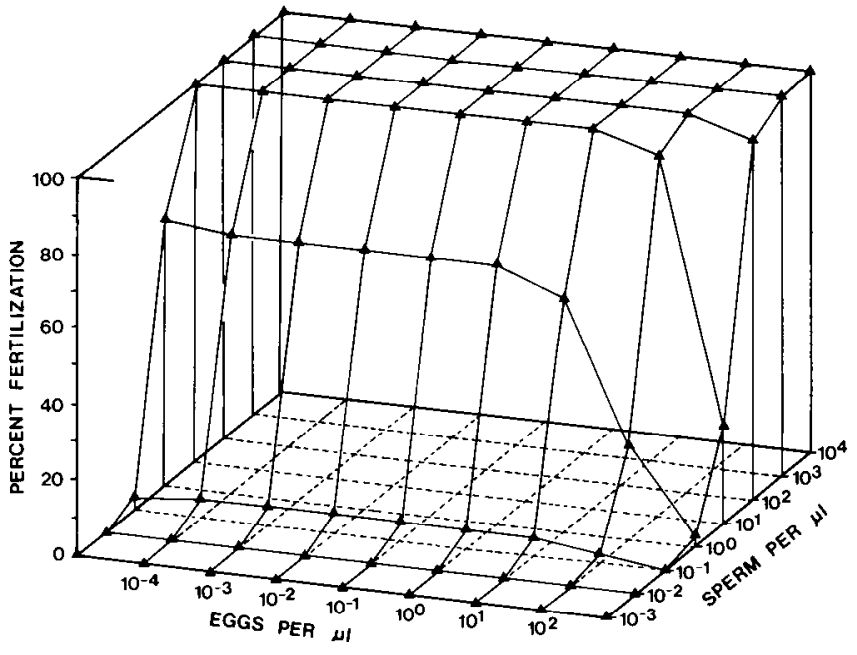

Figure 8. Predicted fertilization as estimated by the VCCW model for a range of sperm and egg concentrations. Sperm are extruded from the gonopore at a concentration of approximately $8.60 \times 10^{6} / \mu \mathrm{l}$. Eggs are extruded at a concentration of approximately $3.25 \times 10^{\gamma} / \mu 1$.

1990; Levitan, 1991). Such a decline in sperm concentration has been shown to cause a profound decrease in fertilization success. Thus, only factors that change before sperm become too dilute to fertilize eggs are of relevance to estimates of fertilization success.

Factors influencing fertilization on the same time scale as sperm dilution are egg concentration and gamete contact time. An additional factor not examined is shear forces on gametes which might further decrease the chance of fertilization in turbulent water (Denny and Shibata, 1989; Denny, pers. comm.). Egg concentration did not significantly affect fertilization success within small containers that would enhance sperm competition. Lillie (1915) also found no significant effect of egg concentration on fertilization with Arbacia. The VCCW model confirms these empirical observations.

Sperm-egg contact time did have a significant effect on fertilization success, particularly within the small time intervals most likely to be important in the field (less than a minute). Rothschild and Swann (1951) came to a similar conclusion with Psammechinus miliaris. Hence it seems evident that in the future, this factor should be incorporated into fertilization models. As sperm and egg plumes expand into the water column, the rate of dilution influences both the concentration of sperm and eggs and the time spent at a particular concentration.

The finding that the lifespan of a spermatozoan is related to concentration has been termed the respiratory dilution effect (Chia and Bickell, 1983). The rate of spermatozoan oxygen consumption increases with dilution; the total amount of oxygen consumed by a spermatozoan over its lifetime, however, is independent of dilution. Thus, a spermatozoan in a concentrated medium can 
maintain a longer lifespan at a lower metabolic level compared to a spermatozoan in a dilute medium. This dilution effect has been attributed to carbon dioxide levels, $\mathrm{pH}$, and possibly levels of cyclic AMP (Chia and Bickell, 1983).

In most cases, sperm longevity would not play a major role in fertilization (Pennington, 1985). However, the increased lifespan of concentrated sperm may be important when gametes are released in low flow conditions (e.g., tide pools). In nature, released gametes have been observed to disperse into the water column (Pearse et al., 1988; Levitan, 1988; pers. obs. MAS, DRL) as well as pooling around the gonopores (Pearse et al., 1988; pers. obs. MAS, DRL). In addition, released sperm can stick together in strings or thin wisps as they travel downstream (pers. obs. MAS, DRL). The increased longevity of released but concentrated sperm might increase the likelihood of fertilization when spawning is not completely synchronous or if the distance between spawning individuals is great.

The above experiments provide information on fertilization kinetics in the laboratory. Estimating fertilization success in the field requires information at the gamete, individual, population, and environmental levels. Gamete level factors can best be studied in the laboratory. Knowledge of gamete kinetics along with cstimates of flow conditions in the field allow for the construction of in situ fertilization models (e.g., Denny and Shibata, 1989). These models can then be compared to field estimates of fertilization success (e.g., Pennington, 1985; Yund, 1990; Grosberg, 1991; Levitan, 1991; Levitan et al., in press). In addition, observations of natural spawning events are needed (e.g., Randall et al., 1964; Pennington, 1985; Giese and Kanatani, 1987; Levitan, 1988; Pearse et al., 1988; Petersen et al., in press), to determine under what demographic and environmental conditions organisms release gametes. A combination of these techniques will ultimately provide a robust understanding of patterns of fertilization success in nature.

\section{Acknowledgments}

We thank S. Lacasse, C. Levitan, and J. Watson for assistance in collecting urchins and the Bamfield Marine Station for providing facilities. Leif Bluck assisted us in the non-linear regression analysis. M. Denny, K. Durante, W. Jaeckle, and T. Rawlings made critical comments on an earlier version of this manuscript. This work was supported by a Bamfield Research Associate Fellowship to DRL, a graduate assistantship from the University of Alberta to MAS, and an NSERC grant to F-SC.

\section{Literature Cited}

Brown, G. G., and J. R. Knouse. 1973. Effects of spcrm concentration, sperm aging, and other variables on fertilization in the horseshoe crab, Limulus polyphemus L. Biol. Bull. 144: 462-470.
Chia, F. -S., and L. R. Bickell. 1983. Echinodermata. Pp. 545-620 in Reproductive Biology of Invertebrates, Volume II: Spermatogenesis and Sperm Function, K. G. Adiyodi and R. G. Adiyodi, eds. John Wiley and Sons, NY.

Denny, M. W. 1988. Biology and the Mechanics of the Wave-swept Environment. Princeton University Press, NJ. 329 pp.

Denny, M. W., and M. F. Shibata. 1989. Consequences of surf-zone turbulence for settlement and external fertilization. Am. Nat. 134: 859-889.

Giese, A. C., and H. Kanatani. 1987. Maturation and spawning. Pp. 252-329 in Reproduction of Marine Invertebrates Vol. 9; General Aspects: Seeking Unity in Diversity, A. C. Giese, J. S. Pearse, and V. B. Pearse, eds. Blackwell Scientific Publication and Boxwood Press, CA.

Gray, J. 1955. The movement of sea-urchin spermatozoa. J. Exp. Biol. 32: $775-801$.

Grosberg, R. K. 1991. Sperm-mediated gene flow and the genetic structure of a population of the colonial ascidian Botryllus schlosseri. Evolution 45: 130-142.

Hultin, E., and B. E. Hagstrom. 1956. The variability in the fertilization ratc. Exp. Cell Res. 10: 294-308.

Levitan, D. R. 1988. Asynchronous spawning and aggregative behavior in the sea urchin Diadema antillarum Philippi. Pp. 181-186 in Echinoderm Biology: Proceedings of the Sixth International Echinoderm Conference, R. D. Burke, P. V. Mladenov, P. Lambert, and R. Parsley, eds. A. A. Balkema Press, Rotterdam.

Levitan, D. R. 1991. Influence of body size and population density on fertilization success and reproductive output in a free-spawning invertebrate. Biol. Bull. 181: 261-268.

Levitan, D. R., M. A. Sewell, and F. S. Chia. in press. How distribution and abundance influences fertilization success in the sea urchin Strongylocentrotus franciscanus. Ecology.

Lillie, F. R. 1915. Studies of fertilization. VII. Analysis of variations in the fertilization power of sperm suspensions of Arbacia. Biol. Bull. 28: $229-251$.

Pearse, J. S., D. J. McClary, M. A. Sewell, W. C. Austin, A. PerezRuzafa, and M. Byrne. 1988. Simultaneous spawning of six species of echinoderms in Barkley Sound, British Columbia. Invert. Reprod. Dev. 14: 279-288.

Pennington, J. T. 1985. The ecology of fertilization of echinoid eggs: the consequence of sperm dilution, adult aggregation, and synchronous spawning. Biol. Bull. 169: 417-430.

Petersen, C. W. 1991. Variation in fertilization rate in the tropical reef fish, Halichoeres bivattatus: correlates and implications. Biol. Bull. 181: 232-237.

Petersen, C. W., R. R. Warner, S. Cohen, H. C. Hess, and A. T. Sewell. in press. Variation in pelagic fertilization rates: implications for production estimates, mate choice, and the spatial and temporal distribution of mating. Ecology.

Randall, J. E., R. E. Schroeder, and W. A. Stark, II. 1964. Notes on the biology of the echinoid Diadema antillarum. Carib. J. Sci. 4: 421-433.

Rothschild, L., and M. M. Swann. 1951. The fertilization reaction in the sea urchin. The probability of a successful sperm-egg collision. J. Exp. Biol. 28: 403-416.

Strathmann, M. 1987. Phylum Echinodermata, Class Echinoidea. Pp. 511-534 in Distribution and Development of Marine Invertebrates of the Northern Pacific Coast, M. Strathmann, ed. University of Washington Press, Seattle.

Vogel, H., G. Czihak, P. Chang, and W. Wolf. 1982. Fertilization kinetics of sea urchin eggs. Math. Biosci. 58: 189-216.

Yund, P. 1990. An in situ measurement of sperm dispersal in a colonial marine hydroid. J. Exp. Zool. 253: 102-106. 